\title{
A Markov Network Based Passage Retrieval Method for Multimodal Question Answering in the Cultural Heritage Domain
}

\author{
Shurong Sheng ${ }^{1}$, Aparna Nurani Venkitasubramanian ${ }^{2}$ and Marie-Francine \\ Moens $^{1}$ \\ 1 Department of Computer Science, KU Leuven \\ 3001 Leuven, Belgium, \\ \{shurong.sheng, sien.moens\}@cs.kuleuven.be \\ 2 Department of Electrical Engineering (ESAT), KU Leuven \\ 3001 Leuven, Belgium, \\ aparna.nuranivenkitasubramanian@esat.kuleuven.be
}

\begin{abstract}
In this paper, we propose a Markov network based graphical framework to perform passage retrieval for multimodal question answering (MQA) with weak supervision in the cultural heritage domain. This framework encodes the dependencies between a question's feature information and the passage containing its answer, with the assumption that there is a latent alignment between a question and its candidate answer. Experiments on a challenging multimodal dataset show that this framework achieves an improvement of $5 \%$ in terms of mean average precision (mAP) compared with a state-of-the-art method employing the same features namely (i) image match and (ii) word co-occurrence information of a passage and a question. We additionally construct two extended graphical frameworks integrating one more feature, namely (question type)(named entity) match, into this framework in order to further boost the performance. The performance has been further improved by $2 \%$ in terms of $\mathrm{mAP}$ in one of the extended models.
\end{abstract}

Keywords: Multimodal Question Answering, Passage Retrieval, Markov Network, Graphical Framework

\section{Introduction}

Multimodal question answering (MQA) refers to answering a textual query on an image. In the cultural heritage domain, MQA enables asking questions on images of cultural artifacts for visitors in museums, landmarks and other sites. This allows a personalized, engaged and natural way for people to interact with cultural relics which, to our best knowledge, doesn't exist yet.

A crucial component of an MQA system is the passage retrieval task that involves the identification of top-ranked passages that may contain the answer for a given question, thereby reducing the search space from a massive collection of documents to a fixed number of passages. Although passage retrieval in opendomain question answering (QA) is widely studied over the last decades, there 


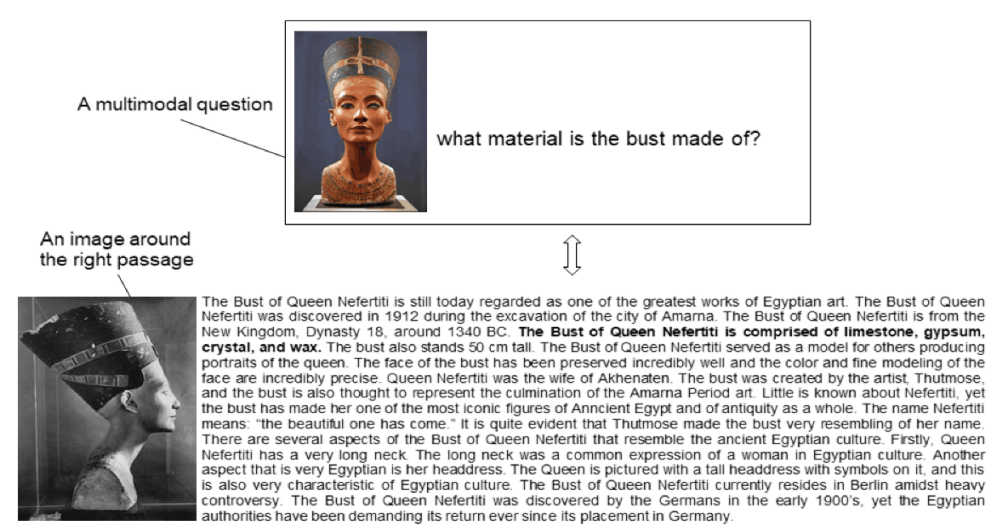

Fig. 1. An exemplary question-passage pair, the answer passage is retrieved by the model proposed in this paper

are few efforts on passage retrieval for MQA in the cultural heritage domain. In this paper, we address the task of retrieving passages (from a multimodal documentation) that contain the answers to a multimodal question. An exemplary question-passage pair is shown in Fig. 1. As in [13], we divide the multimodal questions into two categories: (i) the coarse full-image level questions on the entire image of an artwork, e.g., who is she?, and (ii) the finer partial-image level questions referring to the intricacies of an artwork, e.g., what happened to her left eye?

Compared with traditional passage retrieval in open-domain textual question answering, passage retrieval for MQA in the cultural heritage domain faces several key challenges. First, the multimodal setting injects some uncertainties into the passage retrieval problem, e.g., how to define the relevance of a passage and its associated images in the multimodal documents? How accurate is the definition of this relevance? Second, the text-based resources in the cultural heritage domain are often innately noisy, containing non-standard spelling, poor punctuation, obsolete grammar and word forms [7]. In addition, the information needs and tasks of cultural heritage users are often complex and diverse. For instance, the dataset we use in this paper covers 9 types of diverse questions with some of them being complex. Third, some questions inevitably have no answers due to historical reasons, in which case the questions should be classified as NIL questions, that is, having no answers. As we will see in section 4.1, this may lead to propagated errors for the passage retrieval task in case the questions are wrongly classified. Moreover, partial-image level questions require deep reasoning of both the textual and visual parts of a question.

With the increasing interest in deep learning, huge efforts have been put into both the textual QA task and the MQA task [11,1] employing deep neural networks which need a large amount of question-answer pairs as training data and usually work on sentence-level answers (short answers). Due to the lack of largescale training data and longer answers for MQA in the cultural heritage domain, current end-to-end deep neural networks cannot be directly applied in our task. In this paper, we propose a Markov network based graphical framework encoding 
the dependencies between a question's feature information and the passage containing its answer, with the assumption that there is a latent alignment between a question and its candidate answer. Experimental evaluation in terms of mAP shows that this framework achieves an improvement of $5 \%$ compared with the state-of-the-art proposed in [13] when using the same features namely (i) image match and (ii) word co-occurrence information of a passage and a question.

The main contributions of this paper are as follows: first, we propose and implement a weakly supervised Markov network based graphical framework to perform passage retrieval for MQA in the cultural heritage domain; second, we construct two extended forms of the framework integrating one more feature, namely (question type)-(named entity) match, to further boost the performance with $2 \%$ improvement in terms of mAP.

The remainder of this paper is organized as follows: section 2 introduces related research, section 3 describes the graphical frameworks, section 4 discusses feature extraction experiments and gives an analysis of the final results, section 5 concludes this paper and points out future research.

\section{Related work}

Our work is mainly inspired by [17] that learns entity linking across animal objects of a video and textual mentions from corresponding subtitles. It achieves good results on their cross-modal (vision and language) entity alignment task in a video documentary about wildlife. In contrast to our work, the entity alignment in [17] is more straightforward, e.g., zebra as an object label in the video should be mapped to (ambiguous) mentions of zebra in the text side. While in our task, the alignment for a question and a passage is latent due to their corresponding semantic meaning, requiring a deeper reasoning.

Graphical frameworks are quite popular in performing information retrieval (IR) and question answering $[10,15]$. The difference between these frameworks is the way they encode dependencies in diverse graph structures. [10] focuses on dependencies between different terms while [15] emphasizes the evidence introduced by multiple passages by adding links between several passages sharing common words. In this paper, we encode the dependency of answer passages with feature-level evidence. Furthermore, we encode not only textual clues but also visual evidence i.e., the image match information of a question and a passage in a multimodal setting. [2] also performs passage retrieval in a feature based manner, while they represent features of a question-passage pair as a continuous feature vector, we perform this in a more compact and simple way by embedding them into a graphical framework as discrete variables.

\section{Methodology}

\subsection{Task definition}

Given a multimodal question, we assume that there is a latent alignment in this question and its candidate answers due to some matching features among 
them. That is, the more closely a certain feature matches between a question and a passage, the more likely is the latent alignment between the question and the passage and vice versa. In this paper, we consider feature matches namely image match, word overlap and (question type)-(named entity) match. The first two features are the same as in [13] and the third feature is utilized to further improve the system performance.

This problem is formulated as follows: given a multimodal question $q_{i}$ and a multimodal documentation $D$, a corresponding candidate passage set $P=\left\{p g_{1}, p g_{2}\right.$, $\left.\ldots, p g_{j}, \ldots, p g_{n}\right\}$ needs to be extracted from the documentation $D, n$ is the number of candidate passages; the goal is to retrieve the passages containing the answers to question $q_{i}$; that is, to predict the probability that a passage $p g_{j}$ contains the answer to question $q_{i}$ based on the corresponding feature match information.

For the feature match information, we rely on three sources:

1. Image match between a passage and a question $\left(p g_{j}\right.$. image $\leftrightarrow q_{i}$. image $)$ where a passage image $p g_{j}$.image refers to the image surrounding $p g_{j}$.

2. Word co-occurrence (word_overlap $\left(q_{i}, p g_{j}\right)$ ) which refers to the word tokens occurring in both $q_{i}$ and $p g_{j}$.

The extended graphical models have an additional feature:

3. Question type - named entity match ( $q_{i}$. question_type $\leftrightarrow p g_{j}$.entity). As for certain types of questions in the dataset, i.e., 'Who' and 'Where' questions, e.g., who is this person? where are they from? the answers must contain named entities which are human names and location names respectively.

We address this task using a graphical model that encodes the dependencies between a question $q_{i}$ 's feature match and the candidate passage $p g_{j}$ containing its answer, as described next.

\subsection{Model}

Pairwise Markov random fields (MRFs) forms a subclass of Markov networks which are associated with a set of node potentials and a set of edge potentials. The overall distribution is the normalized product of all the potentials (both node and edge) [4]. A pairwise MRF $G=<\left\{P^{\text {answer }}, P Q^{\text {image }}, P Q^{\text {vec }}\right\}, E>$ is constructed in this paper to judge whether passage $p g_{j}$ is relevant to question $q_{i}$. This MRF $G$ is called basic framework (BF) further in this paper, and it includes the following components:

- A set $P^{a n s w e r}$ of nodes $p_{j} \in\{0,1\}$ in the first row of Fig. 2 (a) with $p_{j}=$ 1 when $p g_{j}$ contains an answer and otherwise 0 . We define a node potential $\varphi_{p j}$ for each $p_{j}$

- A set $P Q^{i m a g e}$ of nodes $p q_{-} i m g_{j} \in\{0,1\}$ in the left part of the second row in Fig. 2(a), denoting the match of passage image $p g_{j} . i m a g e$ and question image $q_{i} . i m a g e$. Here, $\varphi_{p q_{-} i m g_{j}}$ represents the node potential for $p q_{-} i m g_{j}$.

- A set $P Q^{v e c}$ of nodes $p q_{-} v e c_{j} \in\{0,1\}$ in the right part of the second row in Fig. 2(a), denoting the word co-occurrence of passage $p g_{j}$ and question $q_{i}$. $\varphi_{p q_{-} v e c_{j}}$ corresponds with the node potential for $p q_{-} v e c_{j}$. 


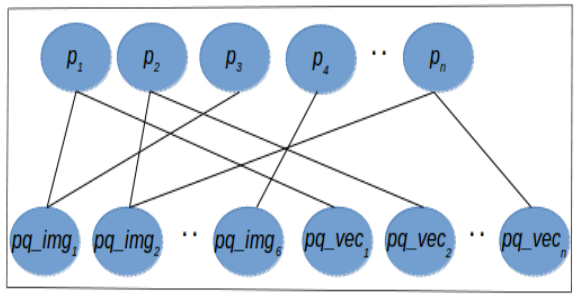

(a)

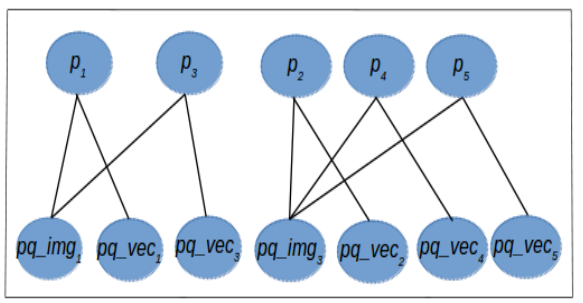

(b)

Fig. 2. (a): Basic framework structure. Each node in the first row refers to a binary variable denoting whether a certain passage contains an answer, and nodes in the second row denote image match and word co-occurrence features between a passage and question a question. The edges between the first and the second row encode corresponding dependencies. (b): Illustration of separated components in the basic framework

$-\forall j$, two edges are built for $p_{j}$ namely, $\left\langle p_{j}, p q_{-} i m g_{j}>\right.$ and $\left\langle p_{j}, p q_{-} v e c_{j}>\right.$. Respective edge potential denoting the dependency weights between $p_{j}$ and its connected node variables are referred to by $\varphi_{\text {edge_pj }}$.

The objective is to maximize the joint probability of all variables for the BF:

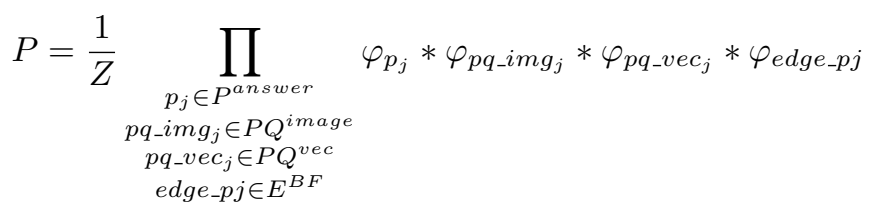

where $Z$ is the normalization constant and $E^{B F}$ is a set of the edges in the BF. The parameters in this equation are initialized as follows:

- As for $\varphi_{p_{j}}$, the potential value for $p_{j}$ being 1 is a prior that allows to incorporate background knowledge (e.g., to indicate that certain passages are inherently related to certain questions). In our experiments, we use an uninformed prior, i.e., $\forall j$, we set $\varphi_{p_{j}}=0.5$ with the assumption that all candidate passages have equal probability to be or not to be the answer to a question.

$-\forall j, \varphi_{p q \_i m g_{j}}$ are assigned in the same way as in [13]: for all candidate passages, the passages in a document right before or after a relevant image of a query image have node potential values of 1 for $p q_{-} i m g_{j}$ being 1 , and this score is then discounted by a fixed number ( 0.2 in our case) which is the same for all documents for further away passages. Details on relevant image extraction are explained in section 4.1 .

$-\forall j, \varphi_{\text {qq }_{-} v e c_{j}}$ is assigned as the cosine score between a question vector and a passage vector when $p q_{-} v e c_{j}$ is 1 . Details on cosine score computation are provided in section 4.2. If a cosine score is 0 , we assign the corresponding potential value to be a very small number near 0 to avoid side effects caused by 0 values.

$-\forall j, \varphi_{e d g e_{-} p j}$ is assigned empirically using a validation set.

Based on the assignments for $\varphi_{p q_{-} i m g_{j}}$, the number of its distinct values is fixed to $M$ (6 in our case, i.e., $1,0.8,0.6,0.4,0.2,0)$. Accordingly, we utilize 
$M$ nodes to denote the image match information in the BF, i.e., the size of $P Q^{\text {image }}$ is $M$. With this property, some passages share the same image match probability, i.e., different passages may be connected due to the shared image match feature. The size of $P^{\text {answer }}$ and $P Q^{v e c}$ equals the number of candidate passages for $q_{i}$. Since each node $p_{j}$ in the first row is only connected to its own feature match information regarding a question $q_{i}$, this framework is composed of several connected components as illustrated in Fig. 2(b).

\subsection{Extended models}

One additional feature (question type)-(named entity) information has been integrated into the two extended frameworks shown in Fig. 3. We call the extended model with two rows in Fig. 3(a) as EF-1 and the other extended model with three rows in Fig. 3(b) as EF-2 further in this paper.

Compared with the BF, EF-1 and EF-2 have an additional set $P Q^{\text {type }}$ of nodes $p q_{-}$type $_{j} \in\{0,1\}$ as shown in Fig. 3. These nodes denote the (question type)-(named entity) match of a question and a passage. Here, $\varphi_{p_{-} q_{-} \text {typ }_{j}}$ represents the node potential of $p q_{-} t_{y p e_{j}}$ and is initialized empirically with pre-defined values according to corresponding match information extracted in section 4.3. There is another set $P^{\text {entity }}$ of nodes entity en $_{k} \in\{0,1\}$ in EF-2 as shown in Fig. 3(b). Each entity $_{k}$ corresponds with an entity entity $k$ in the candidate passages where entity $_{k}$ is 1 when entity_k is the target entity to question $q_{i}$ and 0 otherwise. Here, $\varphi_{\text {entity }_{k}}$ refers to the node potential for entity $y_{k}$. This potential is initialized as 0.5 when entity $y_{k}$ is 1 with the hypothesis that an entity has equal probability to be or not to be the target answer for $q_{i}$. The size of $P^{\text {entity }}$ equals the number of distinct named entities in the whole documentation. We construct an edge between the second and third row if and only if the question type and the named entities of the passage match. For example, if the given question $q_{i}$ is a 'Who' question and passage $p g_{j}$ has and entity $k$ referring to 'PERSON', then there is an edge $\left\langle p q_{-}\right.$type $_{j}$, entity $\left._{k}\right\rangle$. $\varphi_{\text {edge_entity }}$ refers to corresponding edge potential denoting the dependency weights between the node variables in the second and third row in EF-2. Also, since several passages may share some named entities, there may exist loops between the second and the third row.

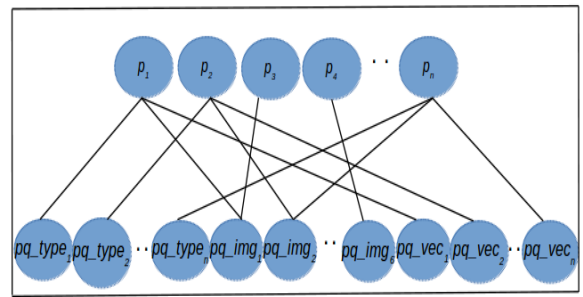

(a)

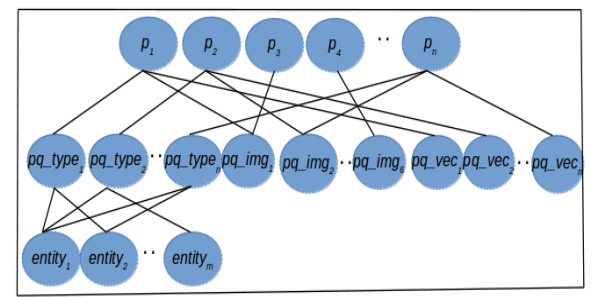

(b)

Fig. 3. (a): Extended framework 1 integrating (question type)-(named entity) match $\left(p_{-}\right.$type $\left._{j}\right)$ in the second row (left part). (b): Extended framework 2 integrating also named entity information in the third row 
The extended models have similar objective functions as the one used in the $\mathrm{BF}$ expressed in equation (1). As for EF-1, the additional $\varphi_{\text {pq }_{-} \text {type }}$ should be added to the right side of equation (1) as an element for the product and both $\varphi_{\text {pq }_{-} \text {type }}$ and $\varphi_{\text {entity }_{k}}$ should be added to equation (1) in the same way as for EF-2.

\subsection{Inference and NIL classification}

If the graphical model has no cycles (e.g., basic framework and EF-1), belief propagation $(\mathrm{BP})$ is employed as the inference method, otherwise when there are cycles (e.g., EF-2), loopy belief propagation (LBP) is applied. In either case, we use the implementation of [12]. Belief propagation works by passing real-valued messages along the edges between connected nodes and LBP starts from an initial set of node messages which are updated until convergence in an iterative manner. This way, the probability that passage $p g_{j}$ contains an answer and the feature match information have mutual influence on each other. Using these inference algorithms, the aforementioned parameters for the three graphical frameworks (namely, edge potential $\varphi_{\text {edge_p }}$ in the BF, all edge potentials in the EF-1, all node and edge potentials in the EF-2) can be validated or updated respectively. The inference method yields a probability list $S_{i}=\left[s_{1}, s_{2}, \ldots s_{j}, \ldots, s_{n}\right]$ in which $s_{j}$ refers to the probability passage $p g_{j}$ containing the answer to question $q_{i}$. As in [13], a threshold $s$ is used to ascertain that a question has NIL as its answer. i.e, $q_{i}$ has NIL as its answer if $\forall j, s_{j}<s$.

\section{Experiments and results}

There are two assumptions for the experiments introduced in this part: first, we benefit from the mature indoor positioning techniques for mobile devices e.g., Bluetooth, Wi-Fi positioning. Under these circumstances, the metadata (id, date, class, etc.) of the artwork regarding a multimodal question can be regarded as prior knowledge since the indoor position of visitors who ask these questions is known. Second, most digital archives of cultural heritage e.g., Europeana ${ }^{3}$, Google Arts \& Culture ${ }^{4}$ support searching by the metadata of an artwork and return its related documents accordingly. Hence, for each artwork in a museum room, its related documents can be regarded as prior knowledge with known metadata.

The feature extraction and model construction are preceded by two preprocessing steps: (i) all tokens in the questions and the documentation are converted to lowercase format; (ii) passages in the related documents concerning the artwork metadata of question $q_{i}$ are extracted, to be added to the graphical model (instead of adding passages from all the documents).

\footnotetext{
3 http://www.europeana.eu/portal/en

4 https://www.google.com/culturalinstitute/beta/
} 


\subsection{Relevant image extraction}

Full-image retrieval: Full-image retrieval is the process of extracting relevant images from the documents, in response to the coarse full-image level questions concerning the entire artifact. We employ manual annotation in this task as in [13], since the related documents for a multimodal question are prior knowledge, the relevant images can then be manually annotated for our small-scale dataset concerning a museum room.

Partial-image retrieval: Partial-image retrieval is the process of extracting relevant image parts from corresponding full images ${ }^{5}$, in response to the finer partial-image level questions concerning only a part of an artwork. In this task, we need to further filter the relevant images for a partial multimodal question by excluding the parts of images that do not concern the partial question image. For example, full relevant images containing the upper body of Akhenaten are relevant for full-image level questions such as 'who is this man?' but not for partial-image question 'why is he barefoot?' whose question image is a photo of Akhenaten's feet. A convolutional neural network (CNN) based method is applied to perform this task wherein the question image is compared with the sub-images detected from the full relevant images using selective search [16]. CNN-based retrieval methods have constantly been proposed in recent years and are gradually replacing the hand-crafted local detectors and descriptors [19]. In this task, we extract the 4096-dimensional descriptor for an input image from the fully-connected (FC7) layer of AlexNet [6] pre-trained on ImageNet [3]. Cosine distance is then utilized to retrieve the relevant partial images for a certain query image. A threshold $(k=0.3)$ is set to filter out irrelevant sub-images. It yields a reasonable retrieval performance with an average accuracy of 0.60 .

\subsection{Word co-occurrence extraction}

In this task, the questions and passages are represented as vectors in a common vector space, where each word represents an axis. A passage is represented as a bag-of-words (bow) as its vector is given by the number of word occurrences (or term frequency, $\mathrm{tf}), p g_{j}=\left[\mathrm{tf}_{1, j}, \mathrm{tf}_{2, j}, \ldots, \mathrm{tf}_{V, j}\right]$, where $V$ is the vocabulary size, i.e., the number of distinct word types in the target collection. A textual query $q_{i}$ is also represented as a $V$-dimensional vector in the same space. We compute the cosine similarity of a query vector and a passage vector to measure the word co-occurrence between them. Stop words are removed and stemming is applied in this procedure.

\subsection{Question classification and named entity extraction}

Question classification: We follow [14] for defining the question types. Question classification refers to classifying all questions into the pre-defined types.

\footnotetext{
${ }^{5}$ These full images are obtained by full-image retrieval described in the beginning of section 4.
} 


\begin{tabular}{|l|c|c|c|c|}
\hline & & Precision & Recall & F1 score \\
\hline \multirow{2}{*}{ Rule-based method } & Who & 0.97 & 0.95 & 0.96 \\
\cline { 2 - 5 } & Where & 1 & 0.9 & 0.94 \\
\hline \multirow{2}{*}{ Logistic regression } & Who & 0.425 & 0.9375 & 0.607 \\
\cline { 2 - 5 } & Where & 0.66 & 0.88 & 0.75 \\
\hline
\end{tabular}

Table 1. Evaluation results for question classification on 'Who' and 'Where' questions

\begin{tabular}{|c|c|c|c|}
\hline & Precision & Recall & F1 score \\
\hline PERSON & 0.87 & 0.87 & 0.87 \\
\hline LOCATION & 1 & 0.9 & 0.94 \\
\hline
\end{tabular}

Table 2. Evaluation results for PERSON and LOCATION type named entities

There are basically two different approaches for question classification: rulebased and machine learning based [5]. We implemented two simple cases of both methods. In the rule-based method, surface words 'who', 'identity', 'name' and 'whose' are applied to detect 'Who' type questions, while the word 'where' is used to detect 'Where' type questions. As for the machine learning based approach, we predict the category of the questions with logistic regression trained on the UIUC dataset ${ }^{6}$. The questions in the training and test set are represented as bagof-words vectors which serve as the input for the logistic regression method. The question type classification in this study is a multi-class classification task. We employed a cross-entropy loss function for logistic regression, using the Scikitlearn library ${ }^{7}$. Since most of the recent learning-based and hybrid approaches use the taxonomy proposed by [8], and the category of the questions in the training set is different from this paper, we need to classify the predicted category to our pre-defined category according to their semantic meaning after the prediction phase.

Table 1 shows the results of these two approaches. Notably, the rule-based method achieves better results in our task. The logistic regression has a relatively lower performance mainly due to the category difference between the training and the test set. Therefore, the rule-based method is utilized to obtain question types.

Named entity recognition: Stanford named entity recognizer (NER) [9] is employed to detect three types of named entities in the passages namely 'PERSON', 'LOCATION' and 'ORGANIZATION'. This NER may classify the same entity to different categories in different documents (based on the context). We employ a majority voting scheme to determine the category label. E.g., if 'Nefertiti' occurs as 'PERSON' 100 times and as 'ORGNIZATION' 50 times, we label it as 'PERSON' in the database. In this task, we assume that the named entity in the cultural heritage domain is not ambiguous, i.e., each named entity belongs to only one category.

The performance of the modified NER introduced in the previous paragraph is evaluated with 100 randomly sampled passages from the documentation as shown in Table 2. Evaluation for 'ORGANIZATION' entities was out of the scope of this paper.

\footnotetext{
${ }^{6}$ http://cogcomp.cs.illinois.edu/Data/QA/QC/

${ }^{7}$ http://scikit-learn.org/stable/modules/generated/sklearn.linear_model.LogisticRegression.html
} 


\begin{tabular}{|c|c|c|c|c|c|}
\hline & & mAP & MRR & NIL Precision & NIL Recall \\
\hline \multirow{3}{*}{ Full } & BF & 0.13 & 0.17 & 0.31 & 0.77 \\
\cline { 2 - 6 } & EF-1 (BF + additional feature) & 0.14 & 0.18 & 0.34 & 0.77 \\
\cline { 2 - 6 } & EF-2 (BF + additional feature) & 0.15 & 0.19 & 0.35 & 0.76 \\
\hline \multirow{2}{*}{ Partial } & BF & 0.091 & 0.091 & 0.67 & 0.77 \\
\cline { 2 - 6 } & BF + partial-image retrieval & 0.092 & 0.092 & 0.67 & 0.77 \\
\hline
\end{tabular}

Table 3. Evaluation results for full-image and partial-image level questions

\begin{tabular}{|c|c|c|c|c|c|c|c|c|c|c|}
\hline & & What & When & Who & Why & Where & Which & How & Yes or no & Selective \\
\hline \multirow{3}{*}{ Full } & BF & 0.13 & 0.06 & 0.13 & 0.09 & 0.04 & 0.02 & 0.5 & 0.19 & 0.06 \\
\cline { 2 - 12 } & EF-1 & 0.13 & 0.06 & 0.13 & 0.09 & 0.07 & 0.02 & 0.5 & 0.19 & 0.06 \\
\cline { 2 - 11 } & EF-2 & 0.13 & 0.06 & 0.12 & 0.09 & 0.19 & 0.01 & 0.5 & 0.18 & 0.10 \\
\hline \multirow{2}{*}{ Partial } & BF & 0.06 & - & - & 0.05 & - & - & - & 0.15 & 0.33 \\
\cline { 2 - 10 } & BF + & 0.06 & - & - & 0.05 & - & - & - & 0.16 & 0.33 \\
\hline
\end{tabular}

Table 4. mAP scores for each type of questions. 'BF+' refers to 'BF + partial-image retrieval' as in Table 3 and '-' means there is no such type of questions

\subsection{Final results of the MQA}

A small validation dataset ${ }^{8}$ is used to estimate the optimal values for parameters in the graphical frameworks and the threshold $s$ for NIL classification introduced in section 3. As for the dataset, there are 807 multimodal questions, 204 multimodal documents and 19587 corresponding paragraphs. Since some passages are filtered out by their constituted entity types in the extended models, the average number of potentially relavant passages per question in the extended models is less than that of the basic framework. This average number of potentially relevant passages per question is 1292 for the $\mathrm{BF}$ and 1109 for the extended models. Corresponding standard deviations of the potentially relevant passage numbers for the two model types are 1364 and 1301 respectively. Other details about the dataset can be found in [14].

There may be several answers to a question, we therefore apply the mean average precision method [13] to evaluate each model's performance. Mean reciprocal rank (MRR) [18] is also employed to evaluate the system's performance to find the top-ranked answer. NIL precision and recall [13] are computed to evaluate the system's ability to detect questions without ground-truth answers.

The evaluation results for the test set $^{9}$ are shown in Table 3. Notably, the $\mathrm{BF}$ achieves a mAP score of 0.13 for the full-image level questions. This is $5 \%$ higher than the best result (0.08) obtained in [13] which employs the same features namely image match and the word co-occurrence as in the BF but uses them sequentially rather than complementarily. After integrating the additional feature namely (question type)-(named entity) match into the BF, the system performance is improved due to the excellent performance in detecting 'Where' questions as shown in Table 3. Here, EF-2 achieves better performance with a $\mathrm{mAP}$ score of 0.15 and a MRR score of 0.19. For partial-image level questions, the system works $1 \%$ better in $\mathrm{mAP}$ score when performing partial-image retrieval.

\footnotetext{
$820 \%$ of the data with 86 question-passage pairs.

${ }^{9} 344$ full-image level questions and 385 partial-image level questions.
} 
The mAP scores shown in Table 4 indicate the system's qualification in answering 'How' type questions in the full-image level question set. With the additional feature, better performance is achieved for 'Where' questions but not in the case of 'Who' questions mainly due to the propagated error of the NIL classification. On one hand some questions having ground-truth answers are wrongly classified as NIL questions with no answers, thus do not contribute to the final mAP score. On the other hand, some NIL questions are classified as questions with ground-truth answers, resulting in an average precision of $0^{10}$.

\section{Conclusion and future work}

In this paper, we have proposed an MRF to perform passage retrieval for MQA in the cultural heritage domain. This framework encodes the dependencies between a question's feature match and the passage containing its answer. We built a basic framework incorporating two features: one textual (word co-occurrence) and one visual (image match) feature. We have also explored extended forms of this framework, by integrating one more feature, i.e., (question type)-(named entity) match. Experiments show that the basic framework improves over the state-of-the-art by $5 \%$ in terms of mAP computed over 344 multimodal questions, and the extended models obtain further improvement with an additional feature.

The graphical models are applied on a challenging small-scale dataset on which it is difficult to train a supervised model. In future work, we will investigate domain adaption methods which can leverage large-scale/external datasets and overcome the limits of the scale of our dataset.

Acknowledgments. This work is funded by the KU Leuven BOF/IF/RUN/2015. We additionally thank our anonymous reviewers for the helpful comments.

\section{References}

1. Andreas, J., Rohrbach, M., Darrell, T., Klein, D.: Neural module networks. In: Proceedings of the IEEE Conference on Computer Vision and Pattern Recognition. pp. 39-48 (2016)

2. Chen, T., Van Durme, B.: Discriminative information retrieval for question answering sentence selection. In: Proceedings of the 15th Conference of the European Chapter of the Association for Computational Linguistics. pp. 719-725 (2017)

3. Deng, J., Dong, W., Socher, R., Li, L.J., Li, K., Fei-Fei, L.: Imagenet: A large-scale hierarchical image database. In: Proceedings of the IEEE Conference on Computer Vision and Pattern Recognition. pp. 248-255 (2009)

4. James, G., Witten, D., Hastie, T., Tibshirani, R.: An introduction to statistical learning, vol. 112. Springer (2013)

$\overline{10}$ This reason is figured out by manually checking the mAP score for each 'Who' question in different models. 
5. Jayalakshmi, S., Sheshasaayee, A.: Question classification: A review of state-of-theart algorithms and approaches. Indian Journal of Science and Technology 8(29) (2015)

6. Krizhevsky, A., Sutskever, I., Hinton, G.E.: ImageNet classification with deep convolutional neural networks. In: Proceedings of the 25th International Conference on Neural Information Processing Systems. pp. 1097-1105 (2012)

7. Lawless, S., Agosti, M., Clough, P., Conlan, O.: Exploration, navigation and retrieval of information in cultural heritage: Enrich 2013. In: Proceedings of the 36th International ACM SIGIR Conference on Research and Development in Information Retrieval. pp. 1136-1136 (2013)

8. Li, X., Roth, D.: Learning question classifiers. In: Proceedings of the 19th International Conference on Computational Linguistics. pp. 1-7 (2002)

9. Manning, C.D., Surdeanu, M., Bauer, J., Finkel, J.R., Bethard, S., McClosky, D.: The Stanford coreNLP natural language processing toolkit. In: Proceedings of the Association for Computational Linguistics (System Demonstrations). pp. 55-60 (2014)

10. Metzler, D., Croft, W.B.: A Markov random field model for term dependencies. In: Proceedings of the 28th Annual International ACM SIGIR Conference on Research and development in information retrieval. pp. 472-479. ACM (2005)

11. Oh, J.H., Torisawa, K., Kruengkrai, C., Iida, R., Kloetzer, J.: Multi-column convolutional neural networks with causality-attention for why-question answering. In: Proceedings of the 10th ACM International Conference on Web Search and Data Mining. pp. 415-424 (2017)

12. Schmidt, M.: UGM: A Matlab toolbox for probabilistic undirected graphical models (2007), https://www.cs.ubc.ca/ schmidtm/Software/UGM.html

13. Sheng, S., Moens, M.F.: Simple baseline models for multimodal question answering in the cultural heritage domain. In: Busch, C., Sieck, J. (eds.) Kultur und Informatik: Mixed Reality, pp. 119-132. Verlag Werner Hülsbusch (2017)

14. Sheng, S., Van Gool, L., Moens, M.F.: A dataset for multimodal question answering in the cultural heritage domain. In: Proceedings of the COLING 2016 Workshop on Language Technology Resources and Tools for Digital Humanities (LT4DH). ACL (2016)

15. Sun, H., Duan, N., Duan, Y., Zhou, M.: Answer extraction from passage graph for question answering. In: Proceedings of the International Joint Conference on Artificial Intelligence. pp. 2169-2175 (2013)

16. Uijlings, J.R., Van De Sande, K.E., Gevers, T., Smeulders, A.W.: Selective search for object recognition. International Journal of Computer Vision pp. 154-171 (2013)

17. Venkitasubramanian, A.N., Tuytelaars, T., Moens, M.F.: Entity linking across vision and language. Multimedia Tools and Applications pp. 1-24 (2017)

18. Voorhees, E.M., et al.: The TREC-8 question answering track report. In: Text REtrieval Conference. pp. 77-82 (1999)

19. Zheng, L., Yang, Y., Tian, Q.: SIFT meets CNN: A decade survey of instance retrieval. arXiv preprint arXiv:1608.01807 (2016) 